\title{
ON UNIFORM OSCILLATION OF THE FIRST AND SECOND KIND
}

\author{
By W. H. Youna, Sc.D., F.R.S.
}

[Received January 4th, 1913.-Read Janua:y 9th, 1913.]

1. The introduction of the concept of uniform convergence marked an epoch in the history of the theory of converging series of functions. If the discovery of far wider conditions which admit of term-by-term integration of series has tended to diminish the importance of the concept in certain branches of the theory, it remains without a rival as affording us a convenient sufficient condition for the continuity of the sum-function of series of continuous functions.

The theory of series has recently undergone important changes. We no longer confine ourselves to converging series, and we have learnt how to distribute all known or knowable functions into classes, and to give simple necessary and sufficient conditions that an assigned function should belong to a particular class.* The question then naturally presents itself as to the possibility of generalising uniform convergence so as to embrace the case of oscillating successions of functions, and at the same time to employ these generalised concepts to obtain sufficient conditions that the upper or lower function of a succession of functions of an assigned type should be of a particular type.

As regards the former desideratum, the generalisation of the concept of uniform convergence, I have already given certain indications. Several modes of generalization are possible, and I have referred to all of them; but their simultaneous use and the adaptation of that portion of the theory I have elaborated, so as to render it available for any lind of functions, are needed. When this has been done the newly devised scheme of classification of functions comes into use and completely justifies its existence by the facility with which it pernits of the application of the tests so obtained.

It is convenient, in the first instance, to suppose the functions of the

"Cf. "On Functions and their Associated Sets of Points," in this volume, pp. 260 seqq. 
succession to be continuous. The upper and lower functions are then necessarily $u l$ and $l u$-functions respectively. We require, then, tests that the upper function may be (i) both a $l u$ and an $u l$, (ii) an $l$, (iii) an $u$, (iv) both an $l$ and an $u$, that is a continuous function, and we require corresponding tests that the lower function should belong to these types.

Now we have this remarkable fact that there are two quite distinct modes of generalising the concept of uniform convergence, ${ }^{*}$ so as to make it applicable to an oscillating succession as a whole. Further, we may so generalise as to require either of these new concepts to apply not only to the succession as a whole, but to each sub-succession; we may, as I have expressed it, require our condition to be homogenous. $t$ Whether we do so or not, and whether we adopt the one or other of the two distinct methods alluded to, we have an irreproachable generalisation of the original concept of uniform convergence, which reduces to it when the upper and lower functions of the succession of continuous functions coincide. When the upper and lower functions do not coincide, each of the two modes furnishes us with tests for distinct properties.

Confining our attention still for convenience to successions of continuous functions, we may say that the succession oscillates uniformly above on the right at the point $x$ in the first mode if the peak function, that is the upper double limit of $f_{n}(x+h)$, as $h$ decreases to zero and $n$ approaches infinity, is at the point $x$ equal to the upper function. If this test is satisfied the upper function is, at the point in question, upper semicontinuous on the right. Similar and corresponding statements, of course, apply to uniform oscillation above on the left, and below on the right and left respectively, in the first mode.

On the other hand, we may say that the succession oscillates uniformly above on the right at the point $x$ in the second mode if, given any quantity $e$, we can find an $n$ and an $h$ so that, for this and all greater values of $n$, and for this and all smaller values of $h$,

$$
f_{n}(x+h)-u(x+h) \leqslant e .
$$

If this test is satisfied the upper function $u(x)$ is at the point $x$ in question lower semi-continuous on the right. 'Corresponding statements apply to uniform oscillation above on the left, and below on the right and left respectively, in the second mode.

*W. H. Young, " Oscillating Successions of Continuous Functions," 1908, Proc. London Math. Soc., Ser. 2, Vol. 6, p. 309 and p. 313. "On Successions whose Oscillation is usually Finite," 1912, Quarterly Journal of Mathematics, Vol. 44, pp. 132, 133, and 141.

$\dagger$ W. H. Young, "On Homogeneous Oscillation of Successions of Functions," 1909, Proc. London Math. Soc., Ser. 2, Vol. 8, p. 353. 
At a point at which the succession of continuous functions oscillates uniformly and homogeneously in the first mode, both above and below, it oscillates uniformly and homogeneously in the second mode, hoth above and below. If throughout an interval the succession oscillates uniformly above in the first mode, it oscillates uniformly above in the second mode except at a set of the second category. The same is true if all that we know of the oscillation above in the first mode at each point is that it is uniform on the right or on the left, but not necessarily on both. Corresponding statements also hold for the oscillation below.

On the other hand, whereas the points of uniform oscillation above, below, on the right, on the left, in the first mode necessarily all exist in the case of a succession of continuous functions except at a set of the first category, this is not the case with those of the second kind, which are, in general, entirely absent.

These remarks enable us to obtain sufficient tests in terms of the uniformity of the oscillation in order that the upper (lower) function should belong to any assigned possible type of function.

When the functions of the succession are no longer continuous the definition above given for uniform oscillation of the first kind is no longer applicable. It appears, however, that we may replace it by a precisely equivalent definition. We have, in fact, only to employ the ascending successions for all values of $m$ of which the general term is the function which is the upper bound of all the functions of the succession from the $m$-th up to the $n$-th inclusive, and the descending succession of which the general term is the corresponding lower bound; the uniform convergence of all the former successions is then taken to be the definition of uniform oscillation above in the first mode, and that of all the second successious the definition of uniform oscillation below in the first mode. Here the convergence and the corresponding oscillation may be considered as to their uniformity either on the right or on the left or both simultaneously.

It then at once suggests itself that the definition of the second mode of uniform oscillation may be modified in a similar manner. It appears, in fret, that, if we take the descending succession of which the general term is the function which is the upper bound of all the functions of the given succession from the n-th onwards, and the ascending succession formed by the corresponding lower bounds, the uniform convergence of the former succession on the right (left) may be taken to be the definition of uniform oscillation above on the right (left) in the second mode, and that of the second succession as that of uniform oscillation on the right (left) below in the second mode. This modification is convenient for various purposes 
but it is not necessary, as it is evident that the original definition is valid for successions of any kind of functions.

We are now able to answer, in terms of the new concepts, questions as to the nature of the upper and lower functions of a succession of functions belouging to any assigned type. For definiteness let us suppose that the

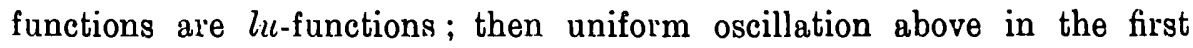
mode has no simplifying effect on the upper function, which is an $u$ lu, but uniform oscillation below in the first mode ensures the lower function being a $l u$ instead of an $u l u$, as it would be in the general case. On the other hand, uniform oscillation in the second mode, when above, makes the upper function a $l u$, and if below makes the lower function an $u l u$, in each case depressing the type by one. If the functions of the successions are all $l u$ 's and $u l$ 's simultaneously, the first mode of uniform oscillation above makes the upper function an $u l$, and uniform oscillation below in the first mode makes the lower function a $l u$. In the second mode these are interchanged, the upper function being a $l u$ and the lower function an $u l$.

These results, in fact, and others of a similar nature follow from the considerations I have just exposed, and the use of the theorem that a uniformly converging sequence of functions of any type has a function of that type for limit, a theorem of which the proof will be found below (\$19).

2. The remarks I have made as to the importance of the concepts of uniform oscillation of the first and second kind are further enforced when we come to adapt an important theorem recently obtained by Egoroff * so as to render it available for oscillating successions. Egoroff finds in terms of the concept of uniform convergence the necessary and sufficient condition that a succession should converge except at a set of content zero. The corresponding problem in oscillating successions is to express in terms of uniform oscillation the necessary and sufficient condition that the upper function should not assume the value $+\infty$, or that the lower function should not assume the value $-\infty$, in each case except at a set of content zero. The result we obtain is as follows :-

It is a necessary and sufficient condition that the upper (lower) finction should be nowhere $+\infty(-\infty)$ except at a set of content zero, that, given any positive quantity e, however snall, we should be able

* D. Egoroff, "Sur les suites de fonctions mesurables," 1911, Comptes Rendus. Seo also "On Successions whose Oscillation is usually Finite," $\$ 4$, loc. cit., supra. 
to find a perfect set whose content differs from that of the fundamental segment by less than e, with respect to which the succession oscillates uniformly above (below) in either the first mode or the second mode or both, as we please.

It is clear that the most convenient test to apply is that one of the three which relates to uniform convergence in the second mode; but all three tests are equally necessary and sufficient.

3. A series of functions is, as is well known, said to be uniformly convergent at the point $P$, if given any quantity $e$, we can find an integer $m$ and an interval $d$ containing the point $x$, so that, for all values of $n \geqslant m$ and all points $x_{1}$ in $d$,

$$
-e \leqslant f_{14}\left(x_{1}\right)-f\left(x_{1}\right) \leqslant e .
$$

Here $m$ and $d$ both depend on $e$, the one in general increasing indefinitely and the other decreasing indefinitely as $e$ approaches zero. We distinguish between uniform convergence pure and simple, uniform convergence on the right, and uniform convergence on the left, according as the interval is unrestricted or has $P$ for left-hand or for right-hand endpoint respectively.

This definition is, when the generating functions $f_{n}(x)$ are continuous, as I have pointed out, precisely equivalent to the following: the series is said to be uniformly convergent at $P$ if, as $n \rightarrow \infty$ and $h \rightarrow 0, f_{n}(x+h)$ has at the point $x$ an unique double limit, this being uniform convergence pure and simple, or uniform convergence on the right (left), according as $h$ assumes all values or only all positive (negative) values.

We may in the case of either definition introduce the distinction between uniform convergence above and below. In the first definition it suffices to divide the double inequality (1) into two inequalities, taking the upper inequality

$$
f_{n}\left(x_{1}\right)-f\left(x_{1}\right) \leqslant e,
$$

to characterize uniform convergence above, and the lower inequality,

$$
-e \leqslant f_{n}\left(x_{1}\right)-f\left(x_{1}\right),
$$

to characterize uniform convergence below. In the case of the second definition, uniform convergence above is determined by the equality of the upper double limit (peak function) of $f_{n}\left(x_{1}\right)$ at the point $x$ with the limiting function $f(x)$, and uniform convergence below by the equality of $f(x)$ and the lower double limit (chasm function) of $f_{n}\left(x_{1}\right)$ at the point $x$. 
The moment, however, we have done this, our two definitions no longer agree. Uniform convergence above defined in the one way does not agree with that defined in the other way, nor does it agree with uniform convergence below defined in the other way. Uniform convergence above in the one way so far agrees with uniform convergence below in the other way that the existence of either property ensures the limiting function being of the same type, the same for both. The one condition, however, is necessarily fulfilled at some point of every perfect set, while the other need not be fulfilled at all.

Moreover, when we proceed to analyse our concept still further and to replace the converging succession of continuous functions by an oscillating one, so that the limiting function has to share its properties between two functions, the upper function and the lower function of the succession, even this point of contact between the two modes of splitting up the concept of uniform convergence into components disappears. We are evidently in the presence of four distinct conceptions, even when we make no difference between right and left, which together make up the equivalent of the single concept of uniform convergence, properly so called.

4. Let us continue to confine our attention to continuous functions in the first instance, and suppose that we have an oscillating succession of such functions. Then we give the following definitions :-

A succession of continuous functions $f_{1}(x), f_{2}(x), \ldots$, is said to oscillate uniformly above on the right in the first mode at the point $x$ if

$$
u(x)=\pi_{R}(x),
$$

where $u(x)$ is the upper function and $\pi_{R}(x)$ the right-hand peak function, that is

$$
\begin{aligned}
u(x) & =\underset{n \rightarrow \infty}{\operatorname{upper}} \operatorname{limit}_{n}(x), \\
\pi_{R}(x) & =\underset{n \rightarrow \infty}{\text { upper double }} \operatorname{limit}_{h \rightarrow 0} f_{n}(x+h) .
\end{aligned}
$$

The same definition applies on the left instead of on the right if we take $h$ to be negative instead of positive, and replace $\pi_{R}(x)$ by $\pi_{L}(x)$. If, on the other hand, we allow $h$ to assume both positive and negative values, and write $\pi(x)$, the peak function, instead of $\pi_{R}(x)$ or $\pi_{L}(x)$, the definition is that of uniform oscillation above in the first mode at the point $x$. Similar definitions apply to uniform oscillations below, using the chasm funtions in place of the peak function and changing upper into lower. Thus, for instance, disregarding the distinction of right and left, we have the definition

A succession of continuous functions $f_{1}(x), f_{2}(x), \ldots$, is said to oscillate 
uniformly below in the first mode at the point $x$ if $l(x)=\chi(x)$, where $l(x)$ is the lower function and $\chi(x)$ is the chasm function, that is

$$
\begin{aligned}
l(x) & =\text { lower limit } f_{n \rightarrow \infty}(x), \\
\chi(x) & =\underset{n \rightarrow \infty}{\text { lower double }} \operatorname{limit}_{n \rightarrow 0} f_{n}(x+h) .
\end{aligned}
$$

In the definition of uniform oscillation of the second kind we need not insert the word "continuous," as the definition, though applied in the first instance to continuous functions, is applicable for all types of functions. The definition is given disregarding the distinction of right and left. If we wish to insert this distinction we only have to restrict $h$ to be positive or to be negative.

A succession of functions $f_{1}(x), f_{2}(x), \ldots$, is said to oscillate uniformly above in the second mode at the point $x$ if, given any positive quantity $e$, we can find an interval $d$ containing the point $x$ as internal point and a corresponding integer $m$, so that for all integers $n \geqslant m$ and all points $x+h$ in the interval $d \quad f_{n}(x+h)-u(x) \geqslant e$,

$u(x)$ being the upper function.

Under the same circumstances if, $l(x)$ being the lower function,

$$
-e \leqslant f_{n}(x+h)-l(x),
$$

the succession is said to oscillate uniformly below in the second mode.

5. Theorex. - If the succession of continuous functions $f_{1}(x), f_{2}(x), \ldots$, oscillates uniformly above (below) in the first mode, the upper function $u(x)$ is upper (lower) semi-continuous.

For, by the definition of uniform oscillation above in the first mode,

$$
u(x)=\text { upper double limit } f_{n}(x+h) .
$$

Now, since any repeated limit is a double limit,

$$
\begin{aligned}
\underset{h \rightarrow 0}{\operatorname{upper}} \operatorname{limit} u(x+h) & =\text { a certain double } \operatorname{limit}_{n \rightarrow \infty} f_{n}(x+h) \\
& \leqslant \text { upper double } \underset{n \rightarrow \infty}{\operatorname{limit}} f_{n}(x+h) .0
\end{aligned}
$$

'I'he comparison of (1) and (2) shows that $u(x)$ is upper semi-continuous. Similarly, since

lower double limit $l(x+h) \geqslant \underset{h \rightarrow 0}{\text { lower double limit }} f_{n}(x+h), \ldots$, the equation defining uniform oscillation below in the first mode, viz.,

$$
l(x)=\text { lower double limit } f_{n}(x+h),
$$

shows that in this case $l(x)$ is lower semi-continuous.

This proves the theorem. 
The inequality (2) of the above proof holds without any special assumption as to the nature of the functions $f_{n}(x)$ or the mode of oscillation of the succession.

The left-hand side of (2) is the associated upper semi-continuous function of the upper function, and the right-hand side may still be defined to be the peak function. Thus we may express (2) by saying that the associated upper limiting function of the upper function is always less than or equal to the peak function. Similarly we may express $\left(2^{\prime}\right)$ by saying that the associated lower limiting function of the lower function is always greater than or equal to the chasm function. If the functions $f_{n}(x)$ are-continuous, (2) remains true when we replace the left-hand side by $u(x)$. The same is, of course, true if $u(x)$ is lower semi-continuous. In the general case, since a function lies between its upper and lower associated semi-continuous functions except at a countable set of points, we have, except at a countable set,

$$
\chi(x) \leqslant l(x) \leqslant u(x) \leqslant \pi(x),
$$

an inequality which has no exceptional points if the functions $f_{u}(x)$ are continuous, or if $u(x)$ is lower semi-continuous and $l(x)$ is upper semicontinuous.

6. Theoreu.-If the succession of continuous functions $f_{1}(x), f_{2}(x), \ldots$, oscillates uniformly above (below) in the second mode, the upper (lower) function $u(x)$ is lower (upper) semi-continuous.

For

$$
\begin{aligned}
& u(x)-u(x+h) \\
& \quad=\left\{f_{n}(x+h)-u(x+h)\right\}+\left\{u(x)-f_{i n}(x)\right\}+\left\{f_{n}(x)-f_{n}(x+h)\right\} .
\end{aligned}
$$

Now, if the $f_{n}$-succession oscillates uniformly above in the second mode, we can find $m$ so that the first term ou the right is less than $e / 3$ for all values of $h$ considered and all values of $n \geqslant m$. Also, since $u(x)$ is one of the limits of $f_{n}(x)$, we can find a value of $n \geqslant m$ for which the second term is less than $e / 3$. Finally, since $f_{n}(x)$ is continuous for this value of $n$, we can find $H$ so that for $h \leqslant H$ the last term is less than $e / 3$. Thus, the lefthand side of the preceding identity is less than $e$, provided $h \leqslant H$. This proves the theorem as far as the oscillation above is concerned. Similarly it may be proved in the alternative case.

Con.-The theorem remains true when the functions $f_{n}(x)$ are not continuous, but only lower (upper) semi-continuous.

In fact, we have not used the upper semi-continuity of $f_{n}(x)$ in the proof of the lower semi-continuity of the upper function nor the lower 
semi-continuity of $f_{n}(x)$ in the proof of the upper semi-continuity of the lower function.

7. Theorear.-If the upper (lower) function is continuous, then the succession of continuous functions $f_{1}(x), f_{2}(x), \ldots$, oscillates uniformly above (below) in both modes or in neither mode.

It will be sufficient to prove the first half of the theorem. Suppose, then, that the succession oscillates uniformly above in the first mode. Then, taking any point $y$, we can find an interval, or tile, $d_{y}$ and an integer $m_{y}$, so that, for all points $x$ in the tile and all integers $n \geqslant m_{y}$,

$$
f_{n}(x) \leqslant u(y)+e .
$$

Hence, if $u(y)$ is continuous at the point $y$ so that we can so choose the tile that for all points $x$ in it

$$
\begin{gathered}
u(y) \leqslant u(x)+e, \\
f_{n}(x) \leqslant u(x)+2 e,
\end{gathered}
$$

which shows that the succession oscillates uniformly above in the second mode.

Again suppose the succession oscillates uniformly above in the second mode; then, corresponding to the point $y$, we can find a tile $d_{y}$ and a corresponding integer $m_{y}$ so that, for all points $x$ in the tile and all integers $n \geqslant m_{y}$,

$$
f_{n}(x) \leqslant u(x)+e,
$$

and, if $u(x)$ is continuous at the point $y$, we can so choose the tile that, for all points $x$ in it,

and therefore

$$
\begin{gathered}
u(x) \leqslant u(y)+e, \\
f_{n}(x) \leqslant u(y)+2 e .
\end{gathered}
$$

Since $e$ is as small as we please this shows that the upper double limit of $f_{n}(x)$ at the point $y$ is not greater than $u(y)$. But, since $u(y)$ is continuous, the upper double limit of $f_{n}(x)$ cannot be less than $u(y)$, as pointed out at the end of $\S 5$. Hence it must be equal to $u(y)$, which shows that the succession oscillates uniformly above in the first mode.

Combining these two results, we see that the theorem is true.

Cor.-Uniform and homogeneous oscillation of the first kind involves uniform and homogeneous oscillation of the second kind.

In the above proof we have not used the continuity of the functions $f_{n}(x)$ except in so far as the definition of uniform oscillation of the first kind is concerned. It follows, therefore, that, whatever type of functions 
generate the given succession, provided only the upper function is continuous, if the succession oscillates uniformly above in the second mode,

$$
u(x)=\pi(x),
$$

and conversely, if this equation holds, the succession oscillates uniformly above in the second mode. Similarly, provided the lower function is continuous, if the succession oscillates uniformly below in the second mode,

and conversely.

$$
l(x)=\chi(x),
$$

8. Before proceeding further it will be convenient to prove certain properties of monotone ascending and descending successions. The following theorem is slightly more general than that usually given.

Theorem.-A monotone ascending succession of l-functions whose limit is continuous converges uniformly. A monotone descending succession of u-functions whose limit is continuous, converges uniformly.

Let $f_{1}(x) \leqslant f_{2}(x) \leqslant \ldots \rightarrow f(x)$, be a monotone ascending succession of l-functions whose limit is continuous. Then, taking any point $x$, we can determine $H$, so that

$$
f(x+h)-f(x) \leqslant e \quad(h \leqslant H) .
$$

Also, since the succession converges at the point $x$, we can find $M$ so that

$$
f(x)-f_{M}(x) \leqslant e .
$$

Also, since $f_{M}$ is lower semi-continuous, we can find $H_{M} \leqslant H$, so that

$$
f_{M}(x)-f_{M}(x+h) \leqslant e \quad\left(h \leqslant H_{M} \leqslant H\right) .
$$

Hence, adding the three inequalities,

$$
f(x+h)-f_{M}(x+h) \leqslant 3 e \quad\left(h \leqslant H_{M}\right) .
$$

But the succession is monotone ascending, therefore

$$
0 \leqslant f(x+h)-f_{n}(x+h) \leqslant 3 e \quad\left(h \leqslant H_{M}, n \geqslant M\right) .
$$

'This proves that at the point $x$ the sequence converges uniformly. Since this is true at every point $x$, this proves the theorem in the first case, and similarly it may be proved in the second case.

Cor.-If a monotone ascending (descending) succession of functions each of which is lower (upper) semi-continuous at the point $P$, has a himiting function which is continuous at the point $P$, the convergence of the succession at the point $P$ is uniforn. 
9. It will be remarked that, in proving the theorem of the last article, we employed the second definition of uniform convergence, and consequently we had only to prove that the lower half of the condition was satisfied in the case of the ascending succession, and the upper half in the case of a descending succession; the other halves are satisfied of themselves. It is of some importance to note that, in the case of ascending successions of continuous functions, it is the lower half of the first definition of uniform convergence which is necessarily satisfied, and it is the upper half in the case of a descending succession. This appears from the following theorem.

Theorem.-If $f_{1} \leqslant f_{2} \leqslant \ldots$ is a monotone ascending succession of functions, the lower double limit of $f_{n}(x+h)$, as $n \rightarrow \infty$ and $h \rightarrow 0$, is equal to the limiting function $f(x)$, at any point $x$ where all the functions $f_{n}(x)$ are continuous.

Since $f_{n}(x)$ has $f(x)$ as unique limit, we can determine $M_{x, e}$ so that, for $n \geqslant M_{x, e}$

$$
0 \leqslant f(x)-f_{n}(x) \leqslant e .
$$

Taking any one of these values of $n$, we can determine $H_{n, x, e}$ so that, for $h \leqslant H_{n, x, e}$,

$$
-e \leqslant f_{n}(x)-f_{n}(x+h) \leqslant e .
$$

Therefore

$$
-e \leqslant f(x)-f_{n}(x+h) \leqslant 2 e \quad\left(n \geqslant M_{x, e}, h \leqslant H_{n, x ; e}\right) .
$$

$A$ fortiori, if $m \geqslant n, \quad f(x)-f_{m}(x+h) \leqslant 2 e$.

Letting $m$ describe such an increasing succession of integers, and $h$ such a monotone descending sequence with zero as limit, that $f_{m}(x+h)$ has its lower double limit $\chi(x)$ as unique limit, we get

$$
f(x)-\chi(x) \leqslant 2 e .
$$

Letting $e$ approach zero, we get from (2),

$$
f(x)-\chi(x) \leqslant 0 .
$$

But, as $e$ approaches zero, $M_{x, e}$ will in general increase without limit, and we may make $n$ in (1) assume values which increase without limit; at the same time $H_{n, x, e}$ will in general decrease down to zero, and we may in any case take in (1) a succession of values of $h$ approaching zero; thus, as $e \rightarrow 0, f_{n}(x+h)$ in (1) approaches a certain double limit, or limits, which are certainly $\geqslant \chi(x)$. Thus we get from the first inequality in (1), by making $e \rightarrow 0$,

$$
0 \leqslant f(x)-\chi(x) .
$$

By (3) and (4),

$$
f^{\prime}(x)=\chi(x) \text {. }
$$


Similarly we have the alternative theorem :-

Theorem.-If $f_{1} \geqslant f_{2} \geqslant \ldots$ is a monotone descending succession of functions the upper double limit of $f_{n}(x+h)$, as $n \rightarrow \infty$ and $h \rightarrow 0$, is equal to the limiting function $f(x)$ at any point $x$ where all the functions $f_{i n}(x)$ are continuous.

10. We now return to oscillating successions of functions and consider the monotone ascending and descending successions associated with it.

The first monotone ascending succession associated with the given succession $f_{1}(x), f_{2}(x), \ldots$, we denote by

$$
v_{1,1}(x) \leqslant v_{1,2}(x) \leqslant v_{1,3}(x) \leqslant \cdots \rightarrow v_{1}(x),
$$

where $v_{1, r}(x)$ is the upper bound of $f_{1}(x), f_{2}(x), \ldots, f_{r}(x)$. There is a countably infinite set of these sequences, the second being defined in the same way from the given succession omitting its first member, and so on, the $r$-th being got by omitting $f_{1}(x), f_{2}(x), \ldots, f_{r-1}(x)$. These monotone sequences lead up to the functions $v_{1}(x), v_{2}(x), \ldots$ of the first monotone descending sequence. Here, of course, $v_{1}(x)$ is the upper bound of the $f_{n}$-succession at the point $x$, and $v_{r}(x)$ is the upper bound of the same, omitting $f_{1}, f_{2}, \ldots, f_{r-1}$. The limit of the monotone descending $v_{r}$-succession is therefore the upper function $u(x)$ of the $f_{n}$-succession.

Thus we have, for the set of the first monotone ascending sequences, and the first monotone descending sequence, the following scheme:-

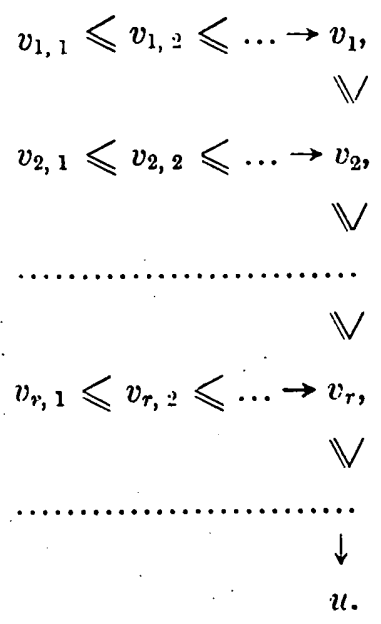

Similarly, taking the lower instead of the upper bounds, we get the sorresponding scheme for the second monotone descending sequences, and 
the second monotone ascending succession associated with the given succession :-

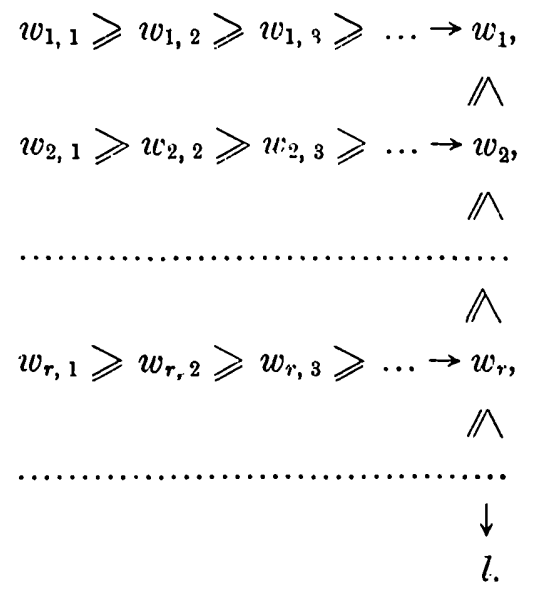

If the functions $f_{n}(x)$ of the given succession are continuous, the same is true of the functions $v_{r, s}(x)$ and $w_{r, s}(x)$. More generally, by one of the fundamental theorems in the theory of the classification of functions, ${ }^{*}$ if all the functions $f_{n}(x)$ are $l$-functions, $l u$-functions, or belong to any other the same class of functions, the same is true of the functions $v_{r, s}(x)$ and $w_{s, s}(x)$.

Hence we can at once give the class to which each of the functions $v_{r}(x), w_{r}(x)$, and $u(x)$ and $l(x)$ belong ; for instance, if $f_{n}(x)$ is continuous, $v_{r}(x)$ is an $l, v_{r}(x)$ is an $u, u(x)$ is an $u l$ and $l(x)$ is a $l u$-function.

11. We now proceed to shew that the uniform oscillation according to the first mode is identical with the uniform convergence of the successions converging to $v_{n}$ and $u_{n}$ for all values of $n$. We shall need the following theorem.

Theorey.-If $f_{1}(x), f_{2}(x), \ldots$ is any succession of functions, and $v_{1}(x) \geqslant v_{2}(x) \geqslant \ldots$, the first associated monotone descending succession, having the upper function $u(x)$ of the $f_{n}$-succession as limit, the peak functions of these two successions are the same, that is to say

upper double $\operatorname{limit}_{n \rightarrow \infty} f_{n \rightarrow 0}(x+h)=$ upper double $\underset{n \rightarrow \infty, h \rightarrow 0}{\operatorname{limit}} v_{n}(x+h)$.

"W. H. Young, "A New Method in the Theory of Integration," 1910, \$6, Proc. London Math. Soc., Ser. 2, Vol. 9, p. 21. 
Writing $\quad \pi(x)=$ upper double $\operatorname{limit}_{n \rightarrow \infty, h \rightarrow 0}$ of $f_{n}(x+h)$,

$$
\pi^{\prime}(x)=\text { upper double limit of } v_{n}(x+h),
$$

we have evidently

$$
\pi(x) \leqslant \pi^{\prime}(x)
$$

since $v_{n}(x+h)$ being the upper bound of $f_{n}(x+h), f_{n+1}(x+h), \ldots$,

$$
f_{n}(x+h) \leqslant v_{n}(x+h) \text {. }
$$

Take such a succession of integers $n$, and such a correspondin $n$ succession of values of $h$, that $v_{n}(x+h)$ has as unique limit its upper double limit. We can then, corresponding to each of these values $v_{n}(x+h)$, take a lesser quantity $V_{n}$, so that, as $n$ describes its succession of values, $V_{n}$ has the same unique limit as $v_{n}(x+h)$, namely $\pi^{\prime}(x)$. Then, corresponding to each of these values of $n$, we can, since $v_{n}(x+h)$ is the upper bound of $f_{n+p}(x+h)$ as $p$ varies, find an integer $m$, depending on $n$, so that

$$
f_{m}(x+h) \geqslant V_{n} \quad(m \geqslant n) .
$$

Now, letting $n \rightarrow \infty$, the left-hand side of the last inequality has a limit, or limits, not exceeding $\pi(x)$; therefore

$$
\pi(x) \geqslant \pi^{\prime}(x)
$$

Hence, by (1),

$$
\pi(x)=\pi^{\prime}(x),
$$

which proves the first statement in the enunciation, that the peak functions of the two successions are the same.

12. Theorem.-The necessary and sufficient condition that a given succession of continuous functions $f_{1}(x), f_{2}(x), \ldots$ should oscillate uniformly above (below) in the first mode, is that the first monotone ascending (descending) successions associated with the given succession should converge uniformly.

First, to show that the condition is sufficient, we assume that the succession of continuous functions

$$
v_{r, 1} \leqslant v_{r, 2} \leqslant \cdots \rightarrow v_{r}
$$

converges uniformly for each integer $r$. Therefore all the functions $v_{r}(x)$ are continuous, so that, by the second theorem of $\S 9$, the limiting function of the $v_{n}$-succession is its peak function. Therefore, by the theorem of $\S 11$,

$$
u(x)=\pi(x) ;
$$

this proves the sufficiency of the condition.

Next, to prove the necessity of the condition, we assume that (1) holds. GER. 2. voL. 12. No. 1181. 
Therefore, since $\pi(x)$ is the upper double limit of $f_{n}(x+h)$, we can find $M>r$ and $H$, depending on $x$ and on $e$, so that for $n \geqslant M$ and $h \leqslant H$,

$$
f_{n}(x+h) \leqslant u(x)+e \leqslant v_{r}(x)+e, \ldots .
$$

Also, since the functions $f_{r}(x), f_{r+1}(x), \ldots, f_{M-1}(x)$ are continuous, we may suppose $H_{M} \leqslant H$ so small that, for $r \leqslant n<M$,

$$
f_{n}(x+h) \leqslant f_{n}(x)+e \leqslant v_{r}(x)+e .
$$

By the last two inequalities

$$
f_{n}(x+h) \leqslant v_{r}(x)+e, \ldots . \quad\left(n \geqslant r, h \leqslant H_{M}\right) .
$$

Hence, since $v_{r}(x)$ is the upper bound of the left-hand side,

$$
v_{r}(x+h) \leqslant v_{r}(x)+e \quad\left(h \leqslant H_{M}\right) .
$$

This shows $v_{r}(x)$ to be upper semi-continuous. But since the functions $f_{n}(x)$ are continuous, $v_{r}(x)$ is necessarily lower semi-continuous $(\S 10)$, and is therefore continuous; therefore, by $\S 8$, the convergence of the monotone succession leading up to $v_{r}(x)$ is aniform. This proves the necessity of the condition as far as the upper oscillation is concerned. Similarly the theorem may be proved for the lower oscillation.

13. It should be remarked that the result of the last article is equally true whether we are considering a particular point or a whole interval. It is also equally true whether we are concerned with uniform oscillation in general or restrict it to be on the right or on the left. The type of oscillation corresponds precisely to the type of the convergence of the corresponding monotone successions. The same remarks apply equally in all similar places in our investigations, and in particular they apply to the theorem which we are about to prove which establishes the connection between uniform oscillation of the second kind and the monotone successions which ascend to the upper and descend to the lower functions.

Thеовем.-The necessary and sufficient condition that a given succession of functions $f_{1}(x), f_{2}(x), \ldots$, should oscillate uniformly above (below) in the second mode is that the second monotone descending (ascending) succession associated with the given succession should converge uniformly to its limiting function, the upper (lower) function of the given succession.

For, since $v_{m}(x)$ is the upper bound of the quantities $f_{m}(x), f_{m+1}(x), \ldots$, the inequality

$$
\begin{array}{lc}
\text { the inequality } & f_{n}(x)-u(x) \leqslant e, \quad(n \geqslant m \geqslant M) \\
\text { has the inequality } \quad v_{m}(x)-u(x) \leqslant e, \quad(m \geqslant M)
\end{array}
$$


as a consequence. Also, since $f_{n}(x)$ cannot be greater than $v_{n}(x)$, the latter inequality entails the former as a consequence. Hence the theorem is true as regards the upper oscillation. Similarly it is true as regards the lower oscillation.

14. The results of the theorems of $\$ \S 12$ and 13 at once furnish us with new proofs of the theorems of $\$ \S 5$ and 6 . They also enable us to obtain with ease a number of further results. Thus it follows from the known theory of uniform convergence of successions of continuous functions that the points at which there is uniform oscillation above, below, on the right, and on the left of the first kind all form sets complementary to sets of the first category, and this equally whether we consider the continuum or any perfect set, always provided that the functions of the succession considered are continuous. We have, indeed, the more general result that this statement is true for the upper oscillation on the right and on the left and in general when the functions of the succession are lower semi-continuous, and that the corresponding statement is true for the lower oscillation when they are upper semicontinuous. In fact, in the former case the functions $v_{1}, v_{2}, \ldots$, are the limiting functions of monotone ascending successions of $l$-functions, and are therefore themselves $l$-functions; hence, bearing in mind that the set composed of a courtable infinite set of sets of the first category is a set of the first category, so that all these $l$-functions $v_{r}(x)$ are continuous except at points of a single set of the first category, the required result follows by means of the corollary to the theorem of $\$ 8$.

Thus we have the theorem :

Theorem.-A succession of lower semi-continuous functions necessarily oscillates uniformly above in the first mode except at a set of points which is of the first category.

Similarly we have the corresponding theorem :

Theorex.-A succession of upper semi-continuous functions necessarily oscillates uniformly below in the first mode except at a set of points which is of the first category.

Both these statements remain, of course, true if we replace the continuum by any perfect set and consider the uniformity of oscillation with respect to this perfect set.

15. There is no corresponding theorem for the points of uniform $2 \triangle 2$ 
oscillation of the second kind. Such points may be entirely absent. We have, however, the following theorem.

Theorem.-When the functions of the succession are continuous and every point is a point of uniform oscillation abone (belove) on one side at least in the first mode, then, except at a set of the first categor!y, there is uniform oscillation above (below) in the second mode.

Take the first of these alternative theorems.

Then the upper bound $v_{n}(x)$ of all but the first $(n-1)$ functions of the given succession, being the limit of a succession of continuous functions which converges uniformly on one side at every point, is a function which is continuous on one side at least at every point, and is, therefore, continuous except at a countable set of points. The upper function $u(x)$ of the original succession, being the limiting function of the monotone descending $v_{n}$-succession, is then a pointwise discontinuous function.* Therefore, except at a set of the first category, consisting of the points of discontinuity of $u(x)$ and the countable set of points at which at least one of the functions $v_{n}(x)$ is discontinuous, all the functions $v_{n}(x)$, as well as the limiting function $u(x)$, are continuous, and therefore the convergence of the $v_{n}$-succession is uniform, so that the original succession oscillates uniformly above in the second mode.

This proves the first of the two alternative theorems. Similarly the other may be proved.

16. We can now answer the question which naturally suggests itself as to sufficient tests that the upper and lower functions of a succession of continuous functions should have an assigned form. It will be sufficient to confine our attention to the upper function, as the corresponding results for he lower function follow immediately. The conditions that the upper function should be upper semi-continuous or lower semi-continuous or both, that is continuous, are obvious. As the upper function is necessarily an ul-function the only remaining condition that we have to investigate is that it should be a $l u$ as well as an $u l$-function. Moreover, since a descending succession of $l$-functions converges to a $l u$-function when the points of uniform convergence exist in every perfect set, a sufficient condition is that there should be no perfect set free from points of uniform oscillation above of the second kind. $†$

* W. H. Young, “On Sequences of Asymmetrically Continuous Functions," 1909. Quarterly Journal, Vol. xL, pp. 374-380.

+ It is sufficient to test the oscillation with respect to each perfect set. 
17. We may also prove the following theorem :-

THeorem.-If the upper (lower) function of a succession of continuous functions is lower (upper) semi-continuous, the points of uniform oscillation above (below) of the second kind form a set complementary to one of the first category.

In fact, the succession $v_{1}(x) \geqslant v_{2}(x) \geqslant \ldots$ leading down to the upper function are in the former of the alternative theorems $l$-functions. Hence, by the argument already used more than once, the statement follows. Similarly the alternative statement follows.

18. We now pass to the consideration of successions of functions other than continuous functions. We have already so far extended our results as to consider the case when the generating functions are upper or lower semi-continuous; but in doing so we only considered those points where these functions are continuous, at least so far as the theory of uniform oscillation of the first kind is concerned. We have up to the present point, in fact, not yet formulated a definition of uniform oscillation of the first kind available at points or in intervals where the generating functions $f_{n}(x)$ of the succession are not continuous.

We now proceed to consider this more general case. We begin by making two remarks. The first is that it follows, from the reasoning of $\$ \S 12$ and 13, that, if a succession of functions converges uniformly, all the monotone successions considered in those articles converge uniformly. This at once suggests that we may replace the definition already given of uniform oscillation of the first kind by that which is identical with it in the case when the functions are continuous, namely, the property proved in $\$ 12$. The second is that the limiting function of a uniformly convergent series of functions of a certain type is always a function of the same type. As this latter remark may not seem obvious, and certainly requires proof, we now give such a proof.

19. TheоReM. - The limiting function of a uniformly convergent succession of functions of a certain type is a function of the same type.

It will be sufficient to treat at length the case when the functions of the succession are $u l$-functions.

Let $e_{1}>e_{2}>\ldots \rightarrow 0$ be any monotone descending sequence with zero as limit. Let us denote some function of the given succession which differs numerically by less than $e_{1}$ from the limiting function $f(x)$, by $f_{1}(x)$, 
another which differs numerically by less than $e_{2}$ from $f(x)$ by $f_{2}(x)$, and so on. This is possible since the convergence is uniform.

Let $G_{1}$ denote the set of points at which

$$
f_{1}(x) \geqslant k-e_{1}
$$

this is an $i$-set, since $f_{1}(x)$ is an $u l$-function.

Let $G_{2}$ denote the set of points at which both

$$
f_{1}(x) \geqslant k-e_{1},
$$

and

$$
f_{2}(x) \geqslant k-e_{2}
$$

this is the common sub-set of two $i$-sets, and is therefore an $i$-set.

Similarly we go on ; thus let $G_{n}$ denote the set of points at which, for $r=1,2, \ldots, n$,

$$
f_{r}(x) \geqslant k-e_{r},
$$

then $G_{n}$ is an $i$-set.

Each of these $i$-sets $G_{n}$ evidently contains the next $G_{n+1}$; they have therefore in common a set $G$, which is the inner limiting set of $i$-sets, and is therefore itself an $i$-set.

At every point $x$ belonging to $G$, we have, for all integers $n$,

$$
f_{n}(x) \geqslant k-e_{n} \text {. }
$$

But $f(x)$ is the limit of $f_{n}(x)$, when $n \rightarrow \infty$ and accordingly $e_{n}$ approaches zero, therefore

$$
f(x) \geqslant k \text {. }
$$

But, on the other hand, if $x$ is a point at which

$$
f(x) \geqslant k,
$$

we must have, since $f_{n}(x)-f(x)$ is numerically less than $e_{n}$,

$$
f_{n}(x) \geqslant k-e_{n},
$$

for all integers $n$, so that $x$ is a point of $G_{n}$ for every $n$, and is therefore a point of $G$. Thus the $i$-set $G$ is precisely the set of points at which

$$
f(x) \geqslant k
$$

which proves that $f(x)$ is itself an $u l$-function.

If the functions $f_{n}(x)$ are $u$-functions, the argument is the same, only instead of $i$-sets, we have closed sets; if they are ulu-functions, we only have $i o$-sets; if ulul-functions, we have ioi-sets, and so on, but in all cases the same argument leads to the required result.

If, on the other hand, the functions $f_{n}(x)$ are $l$.functions, or $l u$-functions, or lul-functions, and so on, we merely have to change the sign $\geqslant$ into $\leqslant$, and the argument applies. In all cases we use the theorem of 
$\$ 24$ of my paper "On Functions and their Associated Sets of Points," which characterises the type of a function by the type of set of points at which it is $\geqslant k$, or $\leqslant k$.

20. We have now to frame a definition of uniform oscillation of the first kind above and below in accordance with the remark of $\$ 18$. A little consideration shows us that the requisite that the ascending succession whose limiting function is $v_{1}(x)$ should be uniformly convergent is precisely equivalent to the following :-

Given $e$, we can find an integer $m_{e}$, so that, whatever point $x$ be taken, there is a corresponding integer $r \leqslant m_{e}$, so that, for all values of $n$,

$$
f_{11}(x)-f_{r_{x}}(x) \leqslant e .
$$

First to show that this is sufficient, suppose the condition to hold. Then, if $p>m_{e}$, the function $f_{r_{r}}(x)$ will, for every point $x$, be among the functions of which $v_{1,2}(x)$ is the upper bound. Hence

$$
f_{n}(x)-v_{1, p}(x) \leqslant e .
$$

But since $v_{1}(x)$ is the upper bound at the point $x$ of $f_{1}(x), f_{2}(x), \ldots$, we can at each point $x$ find an integer $n_{x}$ so that.

$$
f{ }^{\prime}(x) \geqslant v_{1}(x)-e .
$$

Taking $n=n_{x}$ in (1), we get therefore at the point $x$,

$$
v_{1}(x)-v_{1, p}(x) \leqslant 2 e, \ldots \quad\left(p>m_{e}\right) .
$$

Since $m_{c}$ is independent of $x$, and this inequality holds for every point $x$, this shows that the monotone ascending $v_{\mathrm{l}, p}$-succession converges uniformly. Thus the condition is sufficient.

Next to show that it is necessary, we assume that, given $e$, we can find $m_{c}$ so that for all values of $p \geqslant m_{e}$ and all points $x$,

$$
v_{1}(x)-v_{1, p}(x) \leqslant e .
$$

Now $v_{1, p}(x)$ is the upper bound at the point $x$ of $f_{1}(x), f_{2}(x), \ldots, f_{1}(x)$, therefore there is an integer $r_{x}$, such that

$$
v_{1}(x)-f_{r_{x}}(x) \leqslant e, \quad \text { and } \quad r_{x} \leqslant p .
$$

Taking $p=m_{e}$, we see that the given condition is necessary.

Hence we see that the definition of uniform oscillation above in the first mode of a succession of any kind of function $f_{1}(x), f_{2}(x), \ldots$ may be given in the following form :-

The succession $f_{1}(x), f_{2}(x), \ldots$ is said to oscillate uniformly above, in the first way if, given e, we can, corresponding to each integer $r$, find an 
integer $m_{r, e}$, so that, whatever point $x$ be taken, there is a corresponding integer $n_{x} \leqslant m_{r, e}$, so that, for all values of $n$,

$$
f_{r+n}(x)-f_{r+n_{x}}(x) \leqslant e .
$$

The succession is said to oscillate uniformly below in the first mode if,

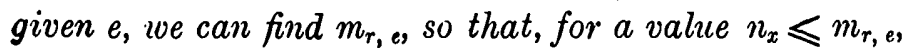

$$
f_{r+{ }_{x}}(x)-f_{r+n}(x) \leqslant e,
$$

for all values of $n$.

This same condition may be expressed as follows:-

The succession $f_{1}(x), f_{2}(x), \ldots$ is said to oscillate uniformly above in the first mode if, given any small positive quantity $e$ and any integer $r$, we can divide the continuum into a finite number $m_{r, e}$ of sets of points, such that, if the point $x$ is any point of the $k-t h$ set,

$$
f_{r+n}(x)-f_{r+k}(x) \leqslant e \quad(n=1,2, \ldots) .
$$

The succession is said to oscillate uniformly below in the first mode if, under the same circumstances,

$$
f_{r+k}(x)-f_{r+n}(x) \leqslant e \quad(n=1,2, \ldots) .
$$

Indeed there is only a finite number of integers $n_{x} \leqslant m_{r, e}$, therefore we may group the points $x$ together in a set, if $n_{x}=k$, where $k$ is any integer $\leqslant m_{r, e}$. If there are no such points, the corresponding set will be the null-set, or, if we prefer, is absent, so that the corresponding integer $k$ will not occur, and the number of sets will be less than $m_{r, e}$.

21. We have now given definitions, in terms of the functions themselves, of uniform oscillation at a point above and below of the first and of the second kinds. The definition of that of the first kind has just been given ( $(20)$, while that of the second kind is the one we originally took. When the fundamental region is the straight line, we may if we please insert the expressions "on the right" or "on the left" with corresponding generalisations for higher space.

We have seen that, when the functions are continuous, uniform oscillation of the first kind always exists except at a set of the first category. The requirement that the succession should oscillate uniformly in the second mode is more stringent, and points of uniform oscillation in the second mode will in general not be present, even when the functions are continuous. When the functions are continuous, however, and the succession is a sequence, uniform oscillation both above and below throughout the continuum of either kind necessitates uniform oscillation 
throughout the continuum of the other kind and ensures the uniformity of the convergence of the original sequence.

When the functions are not continuous it is, however, only the more stringent of the two kinds of uniform oscillation which ensures the uniformity of the convergence of the original sequence, and with it the uniformity of the oscillation in the less stringent of the two modes. To see this last it suffices to suppose the sequence to consist of functions $f_{1}(x), f_{2}(x), \ldots$, of which the odd terms form a monotone descending sequence $f_{1}(x) \geqslant f_{3}(x), \ldots$, and the even terms a monotone ascending sequence $f_{2}(x) \leqslant f_{4}(x) \leqslant \ldots$, both converging non-uniformly to the same limiting function $f(x)$. This is always possible when the functions of the succession are not continuous. But in this case we have clearly uniform and even homogeneous oscillation both above and below of the first kind, combined with non. uniform convergence of the sequence itself.

The justification of the definitions I have given is to be found in the applications, but also in the circumstance, already alluded to, which is fundamental in this connection, that, if a succession converges uniformly, all the monotone successions associated with it do so also, so that the conditions for uniform oscillation, both above and below, of the first and of the second kind are all fulfilled. For completeness I repeat the proof of this fact which I have already given elsewhere.*

22. Theоnem.-If a succession of functions converge uniformly all its associated monotone successions converge also uniformly.

Since the given succession $f_{1}(x), f_{2}(x), \ldots$, converges uniformly we can, after assigning $e$, find $M$, so that, for all values of $m$ and $n$ greater than $M$,

$$
\left|f_{m}(x)-f_{n}(x)\right|<e,
$$

whatever point $x$ be considered, $M$ being independent of $x$. Therefore if

we have, since

$$
M<m<n,
$$

also

$$
f_{n}(x) \leqslant v_{1, m}(x) \text {; }
$$$$
f_{n}(x) \leqslant v_{1, n}(x)+e, \quad(n=m+1, m+2, \ldots)
$$

But $v_{1, n}$ is the upper bound of $v_{1, n}, f_{m+1}, f_{n+2}, \ldots, f_{n}$ at the point $x$. Therefore

$$
v_{1, n} \leqslant v_{1, m}(x)+e, \quad(M<m<n) .
$$

This proves the uniform convergence of the first monotone ascending 
sequence. Similarly the remaining monotone ascending sequences formed from the functions $v_{r, s}(x)$, for successive integers $r$, converge uniformly.

The uniform convergence of the monotone descending $v_{r}$-succession follows as in $\S 13$, since this succession, like the original succession, now has $f(x)$ as limit, and the relations

and

$$
f_{n}(x)-f(x) \leqslant e, \quad(n \geqslant m \geqslant M n
$$$$
v_{n}(x)-u(x) \leqslant e, \quad(n \geqslant M)
$$

are consequences of each other.

This proves the theorem as far as the $v_{r, s}$ and $v_{r}$-successions are concerned. Similarly, making the appropriate changes of ascending into descending, and the like result follows for the $w_{r, s}$ and $w_{r}$-successions.

23. We can now completely answer the question as to the effect of uniform oscillation of the two kinds, above and below, on the right and on the left, on the upper and lower functions of the succession of functions belonging to an assigned class.

'THEOREX.-If the name of the class of functions of a rertain succession begins with an $l$, uniform oscillation of the first kind above prefixes an $u$ to the name of the class in the case of the upper function, so that the class is the same as if the oscillation were not uniform, and, in the case of the lower function, uniform oscillation of the first kind below makes the lower function of the same class as the finctions of the succession. On the other hand, uniform oscillation of the second kind above makes the upper function of the same class as the functions of the succession, while uniform oscillation of the second kind below prefixes an u in the case of the lower function.

Similarly we have the alternative theorem :

Theorear.-If the name of the class of functions of a certain succession begins with an u, uniform oscillation of the first kind above makes the upper function belong to the same class, while uniform oscillation of the first kind below prefixes an $l$ in the case of the lower function, so that the class of the lower function is the same as if the oscillation vere not uniform below. On the other hand, uniform oscillation of the second kind above prefixes an $l$ in the case of the upper function, while uniform oscillation of the second kind below makes the lower function belong to the same class as the functions of the succession. 
The proofs of these theorems will be evident to the reader acquainted with the theory of monotone sequences as exposed in my previously published papers.

From the theorem of $\$ 19$ follows still more directly that if a succession oscillates uniformly above (below) in both ways its upper (lower) function belongs to the same class as the original functions. In fact, the associated monotone successions all converge uniformly, whence the result must be true.

24. We now pass to the generalisation for oscillating successions of the theorem of Egoroff. I have already obtained a generalisation by a method which is itself the extension of Egoroff's method. It is, however, still simpler to assume Egoroff's theorem and to employ the considerations I bave just exposed as to the identity of uniform oscillation with uniform convergence of certain associated monotone successions. If the upper function has the value $+\infty$ at most at a set of zero content, all the monotone successions associated with the upper function are bound to converge except at a set of content zero. Moreover, the convergence, except at a set of content zero, of the successions connected with oscillation of the first kind above, and the convergence, except at a set of content zero, of those connected with oscillation of the second kind above is in either case sufficient to ensure that the points, if any, at which the upper function has the value $+\infty$, form a set of zero content.

Consider first the successions connected with uniform oscillation of the first kind above, namely, the successions of functions $v_{i, s}(x)$. There is a countably infinite number of these successions, each of which converges usually if we suppose, as we shall do, the hypothesis with regard to the upper function to hold. Applying Egoroff's theorem we can separate off a set of content less than $\frac{1}{2} e$ corresponding to the first of these successions, viz., the $v_{1, s}$-succession, a set of content less than $\frac{1}{4} e$ corresponding to the second of these successions, and so on, in such a manner that in the remaining portion of the fundamental region we have uniform convergence of the stccession in question with respect to that portion.

Combining the exceptional sets into a single set of content less than $e$, we have with respect to the complementary set uniform convergence of all the $v_{r, s}$-successions, for every value of $r$.

Similar consideration holds for the lower oscillation. Thus we have the following theorem. 
Theorem.-If the upper (lower) function of a given succession * is $+\infty(-\infty)$ at the points of a set of zero content only, then, given e, we can find a set of content less than $e$ such that with respect to the complementary set the succession oscillates uniformly in the first way. The converse is also true.

In the case of uniform oscillation of the second kind the corresponding result is even more easily perceived, since there is only one monotone succession to be considered.

Theorem.-If the upper (lower) function of a given succession is $+\infty(-\infty)$ at the points of a set of zero content only, then, given e, we can find a set of content less than $e$, such that with respect to the complementary set the succession oscillates above (below) uniformly in the second way. The converse is also true.

25. The results of the preceding article are of great importance, but the reader must be warned not to exaggerate the signification of a theorem such as that of Egoroff. A set even of zero content may be constructed in the continuum in such a manner as to leave over a set of the first category only, $\uparrow$ that is, a set of a kind which does so little towards filling up the continuum that we may subtract a countably infinite number of such sets from the continuum and leave it, in a certain sense, pretty much as it was before. Still more may we say that, when we have been obliged, for the purposes in hand, to remove a set of positive content from the continuum, whether this is a fixed or a variable set, we may have very seriously affected the character of the remaining portion of the continuum from every point of view except that of content. This is illustrated by the fact pointed out by Lusin, that it follows from Egoroff's theorem that any function of any of Baire's classes, and therefore any function of monotone class, is continuous with respect to the complementary set when a suitable set of content as small as we please has been removed from the continuum.

- In the present article the functions of the given succession are supposed finite except at a set of content zero.

+ See, for instance, W H. Young, " On the Construction of a Pointwise Discontinuous Function, all of whose Continuities are Infinities and which has a Finite Generalised Integral," $§ 2$ (1908), Quarterly Journal, Vol. xxxix, p. 218. 